\title{
Formación Inicial docente en Chile y su alineamiento con las temáticas educativas de los Objetivos de Desarrollo Sostenible (ODS)
}

\author{
Rodrigo Andrés MUNDACA GÓMEZ \\ Luis CARRO SANCRISTOBAL
}

Datos de contacto:

Rodrigo Andrés Mundaca

Gómez

Universidad Academia de Humanismo Cristiano rodrigo.mundaca@uacademia.cl

Luis Carro Sancristobal Universidad de Valladolid $\underline{\text { luis.carro@uva.es }}$

Recibido: 28/06/2021 Aceptado: 29/09/2021

\begin{abstract}
RESUMEN
Los Objetivos de Desarrollo Sostenible (ODS) y su agenda educativa 2030, representa los debates y acuerdos que se dan a nivel mundial sobre los compromisos de la humanidad para con nuestra casa común "el planeta". En ese contexto, la formación del profesorado se les reconoce como pilar fundamental para alcanzar una educación de calidad, inclusiva y sostenible para todos, desde un enfoque humanista de la educación y el desarrollo. El foco investigativo se centró en saber cómo se están alineando los proyectos de Formación Inicial Docente (FID) en Chile con relación a las temáticas educativas ODS, para lo cual se abordan tres líneas documentales: la política institucional (PEI, Modelo Educativo); Identidad profesional (Perfiles de egreso); y los planes y programas de estudio. Se sigue procedimientos metodológicos y técnicos propios del paradigma cualitativo basado en la teoría fundamentada. Los resultados muestran, que las temáticas educativas ODS forman parte de las narrativas institucionales, identitarias y programáticas, como contenidos transversales 0 en asignaturas. Sin embargo, su presencia es baja o deficitaria, especialmente en temáticas como el "Desarrollo Sostenible" e "Igualdad de género". Por lo que, se puede concluir que existe un alineamiento en proceso. El supuesto es que serían respuestas adaptativas más que una ruptura con el modelo pedagógico tradicional.
\end{abstract}

PALABRAS CLAVES: formación de profesores; desarrollo sostenible; educación inclusiva; derechos humanos; enfoque de género; democracia. 


\title{
Initial teachers' education in Chile and its alignment with the educational themes of the Sustainable Development Goals (SDG)
}

\begin{abstract}
The Sustainable Development Goals (SDG) and its 2030 educational agenda represents the debates and agreements that take worldwide place on humanity's commitments to our common home "the planet". In this context, teacher training is recognized as a fundamental pillar to achieve quality, inclusive and sustainable education for everyone, from a humanistic approach to education and development. The research was focused on knowing how the "Formación Inicial Docente" (FID) projects in Chile are being aligned with the SDG educational themes, for which three documentary lines are approach: institutional policy (PEI, Educational Model); Professional identity (Graduate profiles) and study plans and programs. There are followed methodological and technical procedures typical of the qualitative paradigm based on grounded theory.

The results show that the SDG educational themes are part of the institutional, identity and program narratives, as transversal contents or in subjects. However, their presence is low or deficient, especially in topics such as "Sustainable Development" and "Gender Equality". Therefore, it can be concluded that there is an alignment in process. The assumption is that they would be adaptive responses rather than a break with the traditional pedagogical model.
\end{abstract}

KEYWORDS: teacher education; sustainable development; inclusive education; human rights, gender equality; democracy.

\section{Introducción}

Los 17 Objetivos de Desarrollo Sostenible (ODS) (UNESCO, 2016), encarnan los compromisos de la humanidad con el planeta y sus ciudadanos. En este escenario, los profesores juegan un rol clave para conseguir sus metas educativas de aquí al 2030 (Portales, 2016), en el entendido que, no se podrán avanzar hacia ellas sino se forman educadores desde una cultura democrática, para la paz y la no violencia (Naciones Unidas, 2015). En esa línea, el interés de esta investigación, se centró en analizar si los proyectos de Formación Inicial Docente (FID) realizados en Chile, se alinean con las temáticas educativas ODS, entre las que se encuentran: sostenibilidad, derechos humanos y ciudadanía, género, enfoque inclusivo y para la diversidad, calidad.

Pero, ¿conocen los docentes los ODS? Martínez (2020) en su investigación concluía que hay bajo conocimiento y escasa formación al respecto, el 87\% de los profesores manifestó no saber de ellos. Otro estudio (Segado Segado et al., 2020) realizado a académicos de una universidad española dicen contar (56\%) con información relativa a los ODS siendo el más conocido el ODS 4, además aseguraban que en sus actividades de docencia los abordan. En Chile, los estudios se centran en su gran mayoría en la llamada educación ambiental o sustentable (Castillo y Cordero, 2019).

En ese contexto, tanto para las universidades como en la FID en Chile, los ODS 
emergen como una oportunidad de redefinir sus perfiles profesionales, estructuras y estrategias políticas (Llanos y Solana, 2016). Respecto a la perspectiva de la sostenibilidad es bajo el número de profesores que cuentan con las competencias para integrarlas a sus prácticas pedagógicas, ni se visualizan en las políticas y marcos normativos proyectos en línea con los ODS. Tampoco se explícita en los programas y planes educativos, de ahí que diferentes investigaciones abogan por su inclusión en el currículum y en su diseño (Opazo et al., 2020; Segado Segado et al., 2020; Vásquez y García-Alonso, 2020; UNESC0, 2017). En tal sentido, educar desde y para el desarrollo sostenible requiere superar las barreras organizacionales en el modo qué se gestionan los proyectos académicos, aún de rasgos individualistas y colaboración fragmentada (de la Rosa Ruiz et al., 2019). En Chile, procesos como la acreditación obligatoria de las pedagogías ${ }^{1}$, estándares orientadores para la FID ${ }^{2}$ y evaluaciones diagnósticas nacionales ${ }^{3}$, entre otras, (MINEDUC, 2016), están llevando a las instituciones a renovar sus programas formativos para estar en sintonía con las nuevas demandas mundiales.

Un estudio realizado por Aznar Minguet et al. (2017) a académicos que forman profesores sostenía que el 53,1\% no considera en sus actitudes y comportamientos saberes de sostenibilidad, sólo el $25 \%$ la incorpora parcialmente y un 35,9\% no las valora o lo hace poco, aunque, un 62,5\%, dice incluirlas en sus actividades curriculares y didácticas. Entre sus causas, estaría el no trabajar de forma interdisciplinar, ni compartir herramientas didácticas. Según Opazo et al. (2020) hay dificultades para desarrollar aprendizajes en contenidos como la paz y los derechos humanos, la diversidad cultural y la igualdad de género, más auspiciosos es encontrar materias relacionadas con el medioambiente o interculturales. En ese contexto, Vilches et al. (2018) proponen integrar los ODS partiendo por una revisión de los currículos, donde saberes de sostenibilidad se trabajen de modo transversal. La demanda es que los futuros profesores se apropien críticamente de competencias profesionales para la sostenibilidad y sus valores, desde acciones reflexivas colaborativas y experienciales, desarrollando capacidades investigativas, metodológicas, didácticas y evaluativas, en el cual se haga visibles buenas prácticas (Aznar Minguet et al., 2017). Por su parte, Opazo et al. (2020) proponen una lectura curricular de los ODS a partir de una "epistemología del sur" que genere quiebres con una "colonización del saber" y en el modo occidental de interpretar el mundo, lo que supone pensar desde una pedagogía de la ruptura y transformadora (Moya Ureta, 2002) y vinculada a saberes comunitarios.

La promesa de no dejar a nadie atrás, que incluye a la educación superior (UNESCO - IIEP, 2017), implica pensar desde una perspectiva holística aquello que tiene que ver con las temáticas educativas ODS y su transversalización en la FID.

En la formación del profesorado contenidos como la democracia, la ciudadanía y los derechos humanos no están del todo incorporados en el currículum, más allá de la importancia retórica que se le asigna por parte de las universidades, en cuanto a

\footnotetext{
${ }^{1}$ Nueva Ley de Educación Superior 20.091 del 2018.

2 Los estándares orientadores (2011) se refieren a los conocimientos disciplinarios y pedagógicos a adquirir una vez finalizado su formación inicial.

${ }^{3}$ Pruebas obligatorias que contempla la ley 20.903 (2016) de desarrollo profesional docente, cuyo objetivo es elaborar planes de mejora de los programas de formativos. 
apropiarse de una cultura democrática y de DDHH, especialmente cuando en la actualidad se constata una caída en el respaldo a la democracia y sus ciudadanos son críticos de ella (Cuenca, 2020). El desafío es superar enfoques academicistas alojados en la educación cívica o en conocimiento de la institucionalidad política (Magendzo y Arias, 2015; Cox, 2018). La salida consiste en generar procesos formativos que las aborden desde un enfoque contextual y de derechos.

Otra de las temáticas más deficitaria en la FID, es aquella que tiene que ver con el enfoque de género. En el estudio de Martínez (2020), además de bajo conocimientos de los ODS, un 97\%, de los consultados afirmaban que hay escasa formación pedagógica que incluya la perspectiva de género. Lo mismo ocurre en Chile, su transversalización en la FID es superficial, hay ausencia de estándares mínimos de calidad que los integren (Sánchez, 2021). Esto se debe a que ha primado una educación sexista que naturalizó las desigualdades de género (Lapierre et al., 2019). Por lo tanto, el reto consiste en formar profesores conscientes de sus prejuicios, y es el deber de la universidad propiciar los espacios e instancias de transformación; incluyendo esta mirada en todo el acontecer educativo y pedagógico, y más directamente en las propuestas e instrumentos curriculares que atañen a la FID (Martínez, 2020; PerrinoPeña y González-Palomares, 2020; Salas Guzmán y Salas Guzmán, 2016;).

En el mismo sentido, el eje clave para alcanzar los ODS es la promoción de una educación inclusiva y para la diversidad. Por lo mismo, la UNESCO (CRES, 2009) sitúa a la educación superior como un bien público estratégico, de primer orden para el mundo democrático desde un enfoque de derechos, justicia social y solidaridad. Lo que implica considerar en las necesidades educativas a todos los estudiantes independiente de su situación socioeconómica, discapacidad, vulnerabilidad social, identidad de género o, si es inmigrante (Lapierre et al., 2019; Valassina et al., 2019).

A nivel de la FID, Martín et al. (2017) explican que hay una aproximación hacia la educación inclusiva a través de contenidos en algunas actividades curriculares, sin embargo, sigue siendo insuficientes, retóricos y lejanos a prácticas pedagógicas concretas (Vélez et al., 2016). Por lo mismo, no es extraño que los profesores declaren no contar con las capacidades para liderar y atender las necesidades educativas de todos sus estudiantes, lo que está poniendo en cuestión los actuales dispositivos de formación (Duk et al., 2019; Herrera-Seda, 2018; Paz Delgado, 2018; Medina Sánchez, 2021; Salas Guzmán y Salas Guzmán, 2016). Fernández Batanero (2013) demuestra que aún falta establecer un tipo de competencias específicas alineadas con la atención a la diversidad o que incluyan herramientas para favorecer la inclusión. En Chile, es posible reconocer buenas prácticas en temáticas de inclusión, aunque la forma ha sido vía normativas, políticas y orientaciones técnico-pedagógicas, implementadas principalmente desde el Estado 4 .

Cervera Delgado y Martí Reyes (2018) refuerzan en sus estudios el planteamiento de que los profesores no se han apropiado del enfoque inclusivo, ni de los recursos para atender la diversidad, debido a que aún navegarían en currículos hegemónicos y

4 Ley 20.845de Inclusión Escolar (2015); Ley N²0.903 de Desarrollo Profesional Docente (2016); Ley 20.911 de Formación Ciudadana (2016); Ley 21.040 de Nueva Educación Pública (2018); Ley 21.091 de Educación Superior (2018). 
técnicos o apoyados en "buenas adecuaciones curriculares". Señalan que esta problemática, no se puede enfrentar solamente con actualizar perfiles y currículos, sino fundamentalmente por comprender y actuar sobre su origen histórico y social. La tarea es levantar políticas públicas que renueven la FID, validando la inclusión y la diversidad como fuente de oportunidades de aprendizaje, ganando influencia en quienes lo diseñan $\mathrm{y}$, a su vez, que permee en todos los documentos e instancias institucionales (Duk et al., 2019; Herrera-Seda, 2018; Medina Sánchez, 2021; Vélez et al., 2016).

En este sentido, la educación inclusiva, como parte del paradigma del desarrollo sostenible, conlleva un nuevo rol e identidad docente, que Paz Delgado resume en las siguientes: "(a) capacidad para desarrollar procesos colaborativos con otros profesores, (b) capacidad para valorar y reflexionar sobre las actitudes, valores y creencias acerca de la diversidad; (c) capacidad para implementar técnicas y estrategias de adaptación de la enseñanza con la finalidad de atender la diversidad del alumnado" (2018, p. 42).

Desde el enfoque de los ODS es esencial redefinir cómo se enseña y aprende en la FID : identidad y género; derechos humanos, democracia y ciudadanía; la inclusión educativa y la atención a la diversidad. Hasta ahora, según Darling-Hammond (2008), la formación inicial ha navegado en un cierto "equilibrio inestable", camino tradicional que para Marcelo y Vaillant (2018) se ha agotado y que, según Escudero (2019), han colocado en tela de juicio su núcleo curricular. La salida, para Magro (2018) y Bolívar (2018), es reprofesionalizar la docencia o hacerla más "disruptiva" donde, al mismo tiempo, se validen sus trayectorias personales y profesionales y, los conocimientos que se aprenden durante toda la vida (Ávalos, 2014; Martín-Romera y García-Martínez, 2018; Serrano Rodríguez y Pontes Pedrajas, 2016).

El desafío es actualizar los proyectos curriculares FID transformando sus procesos de gestión, bajo un nuevo perfil de profesorado capaz de movilizar saberes de distinta naturaleza, conscientes, críticos y comprometidos con lo que le sucede al planeta y a sus ciudadanos.

Para guiar la investigación, se propusieron tres objetivos, a saber:

1. Analizar en los documentos representativos de la política institucional que gestionan la FID en Chile su alineamiento con las temáticas educativas ODS.

2. Analizar el documento perfil de egreso que da identidad profesional a la FID chilena y su alineamiento con las temáticas educativas ODS.

3: Analizar en los documentos programas y plan de estudios de la FID chilena la presencia o ausencia de las temáticas educativas ODS para así medir su nivel de alineamiento.

\section{Método}

El estudio se enmarca en el paradigma cualitativo de tipo descriptivointerpretativo (Rodríguez García et al., 1999). Se focaliza en instituciones chilenas que forman profesores y se analizan documentos bajo criterios metodológicos de un análisis contenido (Noguero, 2002).

Se utiliza el concepto de dimensión para identificar a las tres categorías previas 
objeto de estudio y a la documentación que la acompaña (tabla 1), a saber:

1. Dimensión I política institucional que representa el pensamiento estratégico de las universidades que gestionan la FID (PEI y modelo educativo).

2. Dimensión II identidad profesional que en la FID se exhibe en el texto perfil de egreso.

3. Dimensión III programática referida a los documentos curriculares (planes y programas de estudios) que orientan los proyectos FID considerando sus tres áreas formativas claves (tabla 2).

Del mismo modo se denominan temáticas educativas ODS al conjunto de códigos apriorísticos (Charmaz, 2013) obtenidos de los ODS y que se listan en la tabla 3.

En tal contexto el análisis sistemático documental, supuso el espacio donde se hayan los signos que permiten analizar como la FID universitaria chilena se alinean con las temáticas educativas ODS.

\section{Documentación analizada}

Se eligen cuatro universidades representativas de la FID chilena y cuatro carreras de cada una de ellas (16 pedagogías), dos de primaria y, dos de enseñanza secundaria. Para acceder a los materiales se acude en primer lugar a a los documentos públicos alojados en los portales web en segundo lugar se solicitan vía ley de Transparencia ${ }^{5}$ los documentos perfiles, planes y programas en la tabla 1 y 2 se da cuenta del corpus de datos analizados:

\section{Tabla 1}

Tipo y cantidad de documentos seleccionados por universidad y \% de incidencia

\begin{tabular}{|c|c|c|c|c|c|c|c|}
\hline \multirow[b]{2}{*}{ Universidad } & \multicolumn{7}{|c|}{ Tipo de documento temáticos } \\
\hline & $\begin{array}{c}\text { Proyecto } \\
\text { Educativo } \\
\text { Institucional } \\
\text { (PEI) }\end{array}$ & $\begin{array}{c}\text { Modelo } \\
\text { Educativo }\end{array}$ & $\begin{array}{l}\text { Perfil } \\
\text { de } \\
\text { egreso }\end{array}$ & $\begin{array}{l}\text { Plan de } \\
\text { estudios }\end{array}$ & $\begin{array}{c}\text { Programas } \\
\text { de } \\
\text { asignaturas }\end{array}$ & Total & $\begin{array}{c}\% \text { de } \\
\text { incidencia }\end{array}$ \\
\hline Universidad 1 & 1 & 1 & 4 & 4 & 70 & 80 & 24,1 \\
\hline Universidad 2 & 1 & 1 & 4 & 4 & 88 & 98 & 29,4 \\
\hline Universidad 3 & 1 & 1 & 4 & 4 & 64 & 74 & 22,2 \\
\hline Universidad 4 & 1 & 1 & 4 & 4 & 71 & 81 & 24,3 \\
\hline $\begin{array}{c}\text { Total, } \\
\text { documentos } \\
\text { temáticos }\end{array}$ & 4 & 4 & 16 & 16 & 293 & 333 & 100 \\
\hline
\end{tabular}

Fuente: elaboración propia

5 Ley 20.285 (2009). https://www.portaltransparencia.cl/PortalPdT/web/guest/ley-detransparencia 


\section{Tabla 2}

Número de programas de estudio por carrera, ámbito de formación y \% de incidencia

Número total de Programas de estudio por carrera, ámbito de formación y \% de incidencia

\begin{tabular}{lccccc}
\hline Carrera & $\begin{array}{c}\text { Educativos/ } \\
\text { Pedagógicos }\end{array}$ & $\begin{array}{c}\text { Formación } \\
\text { Práctica }\end{array}$ & $\begin{array}{c}\text { Formación } \\
\text { General }\end{array}$ & $\begin{array}{c}\text { Total, } \\
\text { programas }\end{array}$ & $\begin{array}{c}\% \\
\text { Incidencia } \\
\text { por carrera } \\
25,6\end{array}$ \\
$\begin{array}{l}\text { Educación Parvularia } \\
\text { (EPV) }\end{array}$ & 36 & 24 & 15 & 75 & 25,2 \\
$\begin{array}{l}\text { Educación General } \\
\text { Básica (EGB) }\end{array}$ & 38 & 20 & 16 & 74 & 25,2 \\
$\begin{array}{l}\text { Pedagogía en Historia y } \\
\text { Geografía (HYG) }\end{array}$ & 37 & 22 & 15 & 74 & 24,0 \\
$\begin{array}{l}\text { Pedagogía en Lenguaje y } \\
\text { Comunicación (LYC) } \\
\text { Total, }\end{array}$ & 34 & 21 & 15 & 70 & 100 \\
\% de incidencia & 145 & 87 & 61 & 293 & \\
\hline
\end{tabular}

Fuente: elaboración propia

Se consideró como límite temporal, lo publicado entre los años 2015 y 2019. Respecto a los planes y programas, en algunos casos, son anteriores, pero siguen vigentes por lo cual se incluyen y validan para este estudio.

\section{Instrumentos}

Para la recogida de datos se diseña un conjunto de matrices de análisis a partir de las temáticas educativas ODS (tabla $\mathrm{N}^{\circ} 3$ ) y que se aplican como códigos apriorísticos. En el caso de las dos primeras dimensiones, se construyen matrices favorables a la interrogación de los materiales objeto de análisis (PEI, modelo educativo y perfiles de egreso). Respecto a la dimensión programática, se proponen matrices para codificar los documentos que sostienen el proyecto curricular (plan y programas de estudios) para así determinar la presencia o ausencia de las temáticas en revisión.

\section{Tabla 3}

Temáticas Educativas ODS

Temáticas educativas ODS

$\mathrm{N}^{\circ}$

1. Diversidad e Inclusión

2. Aprendizaje para todos

3. Calidad y Equidad de la Educación

4. Derechos Humanos y Ciudadanía

5. Diversidad cultural 
6. Aprendizaje para toda la vida

7. Desarrollo Sostenible

8. Enfoque de Género

9 Otras (Pobreza, Vulnerabilidad, Solidaridad)

Fuente: elaboración propia

\section{Plan de Análisis}

La estrategia de análisis de contenido (Noguero, 2002), se sostiene en la recopilación constante y sistemática de documentos (tabla 1) y en la práctica del muestreo teórico a través de la codificación (tabla 3), para lo cual se utiliza el software Atlas.Ti 7. Respecto a las dos primeras dimensiones el sistema de codificación y su procesamiento son de carácter inductivo, donde detrás de cada código (o temática ODS) hay una descripción o extracto del documento que valida ese proceso.

Para el caso de la tercera dimensión, el conteo se basa en códigos apriorísticos (temáticas educativas ODS) considerando su nivel de presencia o ausencia en los documentos plan y programas de estudio, se ordenan por ámbitos de formación curricular (ver tabla 2).

\section{Resultados}

\section{Dimensión I Política Institucional}

En su pensamiento estratégico (PEI y modelo educativo) las universidades declaran adherir a una educación humanista, basada en valores como el respeto, pluralismo e inclusión, también en la promoción de la diversidad cultural, social, territorial $\mathrm{y}$, en el compromiso con la excelencia, calidad y equidad educativa distinguiendo a la educación superior como vehículo de movilidad social.

Relacionan la diversidad e inclusión, con abrir sus espacios educativos a los menos favorecidos y a personas con alguna discapacidad adaptando su infraestructura (aula, biblioteca, etc.). Poco es lo que se dice, con relación a la inclusión educativa o atender la diversidad, pero dentro del aula. Para la universidad privada, esta temática, la asocian con ampliar las oportunidades a aquellos con "talento". El uso de tics, aparece como uno de los recursos que favorece generar ambientes inclusivos en contextos de diversidad. Respeto a la diversidad cultural, destacan ideas relativas a la preservación del patrimonio cultural milenario; la integración fronteriza en el ámbito académico; y la valoración de la interculturalidad.

Respecto al aprendizaje para todos y todas, no lo señalan en los PEI. En el documento modelo educativo, indican que cuentan con programas de acompañamiento académico y social, innovan en la docencia y sus currículos se proponen bajo enfoques de competencias. Sin embargo, aún ese proceso radica en los propios estudiantes, en sus desempeños y capacidades ("talentos").

El tema, calidad y equidad de la educación, la relacionan con indicadores de logro funcionales al sistema y, con la instalación de dispositivos de acompañamiento académico como factor de equidad, lo que asocian al alto porcentaje de estudiantes que 
provienen de contextos carenciados, que no contarían con trayectorias escolares favorables a su proceso inicial de formación profesional.

La temática derechos humanos y ciudadanía es parte de su misión, la asocian a prácticas y experiencias de formación vinculadas a la comunidad, una de las universidades regionales incluye como valor humano la integración intercultural.

El aprendizaje para toda la vida, aparece asociado a la formación continua o posgradual, incluye modalidades de enseñanza virtual o b-learning, se ha ido convirtiendo en un nuevo soporte de sustentabilidad económica institucional.

Respecto al desarrollo sostenible o sustentable como lo llaman en Chile, su presencia como temática ODS es baja, lo que aparece está vinculado a la educación ambiental y, al compromiso con el patrimonio cultural. Sólo una de las universidades adhiere a una definición: "el respeto al medioambiente y sus recursos;..."(Universidad 2).

Con relación al enfoque de género, en las narrativas institucionales no se declara explícitamente, esto supone que está presente en otras temáticas como diversidad, inclusión, o derechos humanos. La Universidad 1, señala (una vez) que se fomenta la igualdad de género y sexual.

\section{Dimensión II: Identidad profesional}

En las narrativas que se exponen en los perfiles de egreso hay un alineamiento con las temáticas educativas ODS.

En diversidad e inclusión, exponen conceptos como inclusivo, integral, reflexivo, colaborativo, empático, innovador, articulado $\mathrm{y}$, lúdico, incorporación familia y comunidad, entre otros:

"Implementa estrategias de trabajo colaborativo, para atender a los desafíos propios de su acción docente, demostrando empatía, compromiso con el aprendizaje, valoración de la diversidad y respeto por las diferencias individuales de cada sujeto..." (Perfil_EPV_U1)

"Maneja conocimientos y estrategias para el trabajo con la familia, y la comunidad..."

..."Respeta la diversidad considerándola en la toma de decisiones inclusivas del currículo educacional (Perfil_EPV_U2)

En las pedagogías de educación secundaria se enuncia en saberes disciplinares más cercano a una definición de diversidad cultural:

"Conoce comprende y transforma la diversidad cultural, las tradiciones y el desarrollo histórico del contexto de intervención en una oportunidad de desarrollo didáctico de primer orden." (Perfil_HYG_U2)

En los perfiles declaran formar profesores para desempeñarse en "contextos diversos", asumiendo una postura crítica y creativa, donde desarrolla competencias para la vida y la promoción de la participación de los jóvenes en su entorno.

La temática aprendizaje para todos y todas se materializa en prácticas formativas situadas de mejoramiento curricular, metodológico, didáctico y evaluativo, vinculadas al contexto educativo, social y cultural:

"Aplica metodologías que favorezcan el aprendizaje integral y acorde a los ciclos formativos." (Perfil_EPV_U2) 
"Para ello el/la profesor/a en formación ... generar propuestas didácticas tuteladas, crear oportunidades de aprendizaje significativo para sus estudiantes, diseñar estrategias colaborativas para su implementación... (Perfil_HYG_U1)

También, por la capacidad para saber gestionar oportunidades de aprendizaje para todos, realizando adaptaciones al currículo, haciendo uso de diferentes recursos didácticos, estrategias y medios educativos, desde una visión integral, innovadora y actualizada del fenómeno educativo:

"Demuestra habilidades de liderazgo en el fortalecimiento de una cultura escolar colaborativa para lograr el aprendizaje y el desarrollo personal y social de todos los estudiantes ..." (Perfil_HYG_U3).

"Desarrolla situaciones de enseñanza aprendizaje de lengua, literatura y comunicación, considerando diversos contextos educativos para el logro de una pertinente transposición didáctica." (LYC_U2)

El aprendizaje para todos, desde lo expuesto, se resume en tres ideas: liderazgo pedagógico (crítico, analítico, reflexivo, autónomo y, emocional); desarrollo del pensamiento; y la instalación de una cultura escolar colaborativa.

En materia de derechos humanos y ciudadanía se asumen principios humanistas, éticos y valóricos de responsabilidad social con los contextos vulnerables y multiculturales:

" Comprometido con la educación de ciudadanos, evidencia valores humanos, como la formación personal y social, comunicación, relación con el medio natural y cultural, el respeto por los demás, la valoración e inclusión plena a la diversidad de la región y el país." (Perfil_EGB_U4).

“... una visión humanista centrada en el respeto a la diversidad, con un gran sentido de sensibilidad y responsabilidad social, ..., manifiesta y vivencia la valoración por la persona humana en su integralidad más profunda." (Perfil_HYG_U2)

Se pudo constatar, una formación que se alinea con una cultura escolar democrática y colaborativa, dialógica e inclusiva, que potencia espacios de ciudadana y liderazgo pedagógico en las escuelas y sus comunidades, respetando la diversidad de la región y el país.

Respecto a la educación de calidad con equidad, expresan:

"gestiona sistemas educativos para el logro de la calidad y la excelencia basado en el conocimiento del currículum nacional, la evaluación y la didáctica como herramienta clave en el diálogo docente estudiante." (Perfil_LYC_U4)

“... se caracteriza por demostrar competencias genéricas trasversales, como el compromiso con la sociedad, la orientación a la excelencia, el mejoramiento continuo y la comunicación profesional."(EPV_U4)

"Reflexiona críticamente sobre el rol docente, a través de la investigación en la propia práctica y la articulación de los saberes pedagógicos.” (Perfil_EGB_U1) 
De acuerdo con lo expresado, entre los factores que se alinean con una educación de calidad y equitativa se encuentran: la articulación de saberes de la formación; una mejora permanente de las propuestas pedagógicas y su contextualización; el trabajo colaborativo con sus comunidades educativas; el desarrollo de capacidades y habilidades de indagación, reflexión crítica y de responsabilidad profesional y compromiso social; $\mathrm{y}$, en el cumplimiento a las demandas del sistema educativo.

El aprendizaje para toda la vida lo vinculan con instalar una cultura del aprendizaje como proceso continuo en estrecha relación con su formación pedagógico-disciplinar, pero también, a saberes transversales como: el manejo de un segundo idioma; el uso eficiente de recursos tecnológicos; investigar de manera crítica sobre su propia práctica; en la apropiación de estrategias de enseñanza - aprendizaje innovadoras y colaborativas; $y$, aprendiendo a reconfigurar su conocimiento disciplinar:

"Ser un investigador permanente de su propio que hacer, en la búsqueda continua del mejoramiento de su acción de educador..." (Perfil_EPV_U3).

"Problematiza reflexivamente su quehacer profesional favoreciendo la construcción del saber docente como un proceso continuo y permanente." (Perfil_EGB_U2)

"Asimismo, en su práctica pedagógica, ..., compromiso social e interés en la formación permanente (Perfil_LYC_U1)

“... establece relaciones de trabajo colaborativo con los actores de la comunidad educativa, ... (Perfil_EPV_U1)

Validan el trabajo y aprendizaje con otros profesionales, de manera colaborativa, creando comunidades docentes, profundizando en saberes didácticos, replanteándose y problematizando reflexivamente sobre su quehacer profesional de modo permanente.

Respecto a enfoque de género, no hay una narrativa que recoja esta perspectiva. $\mathrm{Al}$ igual que en la primera dimensión, el supuesto es que se integran como subtemas en otras temáticas educativas ODS. En la carrera de EPV y EGB es normal hablar de niños o niñas, sólo en pedagogía en Historia y Geografía de la Universidad 1, hay un intento de uso de un lenguaje de género, tal como lo señalan:

“..., lo que permitirá que las y los estudiantes emitan juicios críticos autónomos, ...." (Perfil_HYG_U1)

La temática educativa desarrollo sostenible, se presentan como contenido transversal y en asignaturas. En EPV, aparecen en ámbitos de la formación personal social, proyectos de vida saludable, comunicación y en contenidos relacionadas con el medio natural y cultural:

"Considera los saberes propios de la salud infantil: nutrición, puericultura, primeros auxilios para ejecutar proyectos de vida saludable." (Perfil_EPV_U2)

En la universidad 2, en pedagogía en Historia, se presenta como contenidos relativos a la sustentabilidad (ambiental, ecológica, y cultural).

“... promueve en sus estudiantes una vida respetuosa del medio ambiente y comprometida con el mejoramiento y la equidad social..."; “ ..., aplicando criterios de análisis..., como la ecología, diversidad, inclusión, marginalidad,...," 
“... hacer de sus estudiantes sujetos activos y responsables ante el desafío de la convivencia democrática y el desarrollo sustentable" (Perfil_HYG_U2).

En pedagogía en "Lenguaje y Comunicación" no se refieren a esta temática.

\section{Dimensión III: Programática}

Lo que se expone es la síntesis que agrupa en un mismo análisis a las 4 carreras estudiadas es decir 16 pedagogías, el corpus de datos se expuso en la tabla 2.

Se revisaron 4 planes de estudios y 292 programas de asignatura: 145 (50\%) en el ámbito educativo/pedagógico; 87 (30\%) en formación práctica; y 60 (20\%) de formación general. Por carrera, se revisan: 75 programas $(25,7 \%)$ en Educación Parvularia (EPV); 73 (25\%) en Educación General Básica (EGB); 74 (25\%) en Historia y Geografía (HYG); y 70 (24\%) en Lenguaje y Comunicación (LYC).

En ese escenario la dimensión analiza la presencia o ausencia de las temáticas educativas ODS en los planes y programas de estudios por ámbito formativo, sus resultados se exponen en las tablas 4,5 y 6.

\section{Ámbito educativo/pedagógico}

\section{Tabla 4}

Presencia/ausencia de temáticas educativa ODS: Una visión de conjunto, ámbito educativo/pedagógico

Temáticas educativas ODS

CARRERAS

(Presencia/Ausencia)

EPV EGB HYG LYC Promedio

\begin{tabular}{lcccccccccc} 
& $\% \mathrm{P}$ & $\% \mathrm{~A}$ & $\% \mathrm{P}$ & $\% \mathrm{~A}$ & $\% \mathrm{P}$ & $\% \mathrm{~A}$ & $\% \mathrm{P}$ & $\% \mathrm{~A}$ & $\% \mathrm{P}$ & $\% \mathrm{~A}$ \\
\hline Diversidad e Inclusión & 72 & 28 & 63 & 37 & 57 & 43 & 43 & 57 & 59 & 41
\end{tabular}

educativa

$\begin{array}{lllllllllll}\text { Aprendizaje para todos y todas } & 42 & 58 & 45 & 55 & 41 & 59 & 43 & 57 & 43 & 57 \\ \text { Calidad y Equidad de la } & 47 & 53 & 47 & 53 & 35 \% & 65 & 51 & 49 & 45 & 55\end{array}$

Educación

$\begin{array}{lllllllllll}\text { Derechos Humanos y } & 22 & 78 & 13 & 87 & 35 & 65 & 40 & 60 & 28 & 73\end{array}$

Ciudadanía

$\begin{array}{lllllllllll}\text { Diversidad cultural } & 44 & 56 & 55 & 45 & 43 & 57 & 20 & 80 & 41 & 60\end{array}$

$\begin{array}{lllllllllll}\text { Aprendizaje para toda la vida } & 17 & 83 & 8 & 92 & 24 & 76 & 26 & 74 & 19 & 81\end{array}$

$\begin{array}{lllllllllll}\text { Desarrollo Sostenible } & 33 & 67 & 45 & 55 & 49 & 51 & 51 & 49 & 45 & 56\end{array}$

$\begin{array}{lllllllllll}\text { Enfoque de Género } & 8 & 92 & 16 & 84 & 5 & 95 & 14 & 86 & 11 & 89\end{array}$

$\begin{array}{llllllllllll}\text { Otras (Solidaridad) } & 25 & 75 & 3 & 97 & 30 & 70 & 34 & 66 & 23 & 77\end{array}$

$\begin{array}{lllllllllll}\text { Promedio General por carrera } & 35 & 65 & 33 & 67 & 35 & 65 & 36 & 64 & 35 & 65\end{array}$

Fuente: elaboración propia

Como se consigna en la Tabla 4, ninguna de las temáticas educativas ODS supera el $35 \%$ de presencia en los programas. Al respecto, "Igualdad de género" (89\%), "aprendizaje para toda la vida" (81\%), "Derechos Humanos y Ciudadanía “(73\%) 
muestra un déficit de presencia o poco alineamiento con los ODS, de acuerdo con los resultados aún se estaría lejos de alcanzar un currículum que incorpore a sus procesos el paradigma de la sostenibilidad y su perspectiva humanista e inclusiva de la educación.

\section{Ámbito formación práctica}

\section{Tabla 5}

Presencia/ausencia de temáticas educativa ODS: Una visión de conjunto, ámbito Formación práctica

\begin{tabular}{|c|c|c|c|c|c|c|c|c|c|c|}
\hline \multirow[t]{3}{*}{ Temáticas educativas ODS } & \multicolumn{8}{|c|}{$\begin{array}{c}\text { CARRERAS } \\
\text { (Presencia/Ausencia) }\end{array}$} & & \\
\hline & \multicolumn{2}{|c|}{ EPV } & \multicolumn{2}{|c|}{ EGB } & \multicolumn{2}{|c|}{ HYG } & \multicolumn{2}{|c|}{ LYC } & \multicolumn{2}{|c|}{ Promedio } \\
\hline & $\% \mathrm{P}$ & $\% \mathrm{~A}$ & $\% \mathrm{P}$ & $\% \mathrm{~A}$ & $\% \mathrm{P}$ & $\% \mathrm{~A}$ & $\% \mathrm{P}$ & $\% \mathrm{~A}$ & $\% \mathrm{P}$ & $\% \mathrm{~A}$ \\
\hline $\begin{array}{l}\text { Diversidad e Inclusión } \\
\text { educativa }\end{array}$ & 63 & 38 & 60 & 40 & 59 & 41 & 62 & 38 & 61 & 39 \\
\hline $\begin{array}{l}\text { Aprendizaje para todos y } \\
\text { todas }\end{array}$ & 79 & 21 & $75 \%$ & 25 & 32 & 68 & 29 & 71 & 54 & 46 \\
\hline $\begin{array}{l}\text { Calidad y Equidad de la } \\
\text { Educación }\end{array}$ & 33 & 67 & $30 \%$ & 70 & 50 & 50 & 57 & 43 & 43 & 58 \\
\hline $\begin{array}{l}\text { Derechos Humanos y } \\
\text { Ciudadanía }\end{array}$ & $25 \%$ & $75 \%$ & $10 \%$ & $90 \%$ & $5 \%$ & $95 \%$ & 10 & 90 & 13 & 88 \\
\hline Diversidad cultural & 67 & 33 & 60 & 40 & 86 & 14 & 10 & 90 & 56 & 44 \\
\hline $\begin{array}{l}\text { Aprendizaje para toda la } \\
\text { vida }\end{array}$ & 38 & 63 & 35 & 65 & 73 & 27 & 0 & 100 & 37 & 64 \\
\hline Desarrollo Sostenible & 38 & 63 & 45 & 55 & 5 & 95 & 86 & 14 & 44 & 57 \\
\hline Enfoque de Género & 13 & 88 & 25 & 75 & 0 & 100 & 52 & 52 & 23 & 77 \\
\hline Otras (solidaridad) & 0 & 0 & 0 & 0 & 5 & 95 & 38 & 62 & 11 & 39 \\
\hline $\begin{array}{l}\text { Promedio General por } \\
\text { carrera }\end{array}$ & 44 & 56 & 43 & 58 & 35 & 65 & 62 & 38 & 46 & 54 \\
\hline
\end{tabular}

Fuente: elaboración propia

En este ámbito, la temática con más presencia es "diversidad e inclusión educativa" con un $61 \%$, le siguen "diversidad cultural" y "aprendizaje para toda la vida" con un 56\%. Por carrera, "Aprendizaje para todos" en EPV y EGB están presentes en un 70\%, sin embargo, en Historia y Lenguaje no superan el 30\%. Esto, se puede deber, en parte, a que son ciclos educacionales distintos (niños y jóvenes), en secundaria, por lo general el foco está puesto en lo disciplinar. Dos casos más: Desarrollo Sostenible llega al 44\% e "Igualdad de Género" al 23\%, esto significa que en los diseños curriculares su presencia es al menos deficitaria.

Los resultados expuestos en la tabla 5, coinciden con el ámbito anterior, todas las temáticas educativas ODS están bajo el 45\%, más del 54\% de los programas revisados no las integran a sus proyectos académicos, lo que muestra un débil alineamiento de los currículums en este ámbito, sólo LYC alcanza mayor presencia (62\%). 


\section{Ámbito formación general}

\section{Tabla 6}

Presencia/ausencia de temáticas educativa ODS: Una visión de conjunto, ámbito Formación general

\begin{tabular}{|c|c|c|c|c|c|c|c|c|c|c|}
\hline \multirow[t]{3}{*}{$\begin{array}{l}\text { Temáticas } \\
\text { educativas ODS }\end{array}$} & \multicolumn{10}{|c|}{$\begin{array}{c}\text { CARRERAS } \\
\text { (Presencia/Ausencia) }\end{array}$} \\
\hline & \multicolumn{2}{|c|}{ EPV } & \multicolumn{2}{|c|}{ EGB } & \multicolumn{2}{|c|}{ HYG } & \multicolumn{2}{|c|}{ LYC } & \multicolumn{2}{|c|}{ Promedio } \\
\hline & $\% \mathrm{P}$ & $\% \mathrm{~A}$ & $\% \mathrm{P}$ & $\% \mathrm{~A}$ & $\% \mathrm{P}$ & $\% \mathrm{~A}$ & $\% \mathrm{P}$ & $\% \mathrm{~A}$ & $\% \mathrm{P}$ & $\% \mathrm{~A}$ \\
\hline $\begin{array}{l}\text { Diversidad e } \\
\text { Inclusión } \\
\text { educativa }\end{array}$ & 13 & 87 & 7 & 93 & 7 & 93 & 7 & 93 & 9 & 92 \\
\hline $\begin{array}{l}\text { Aprendizaje } \\
\text { para todos y } \\
\text { todas }\end{array}$ & 7 & 93 & 0 & 100 & 0 & 100 & 0 & 100 & 2 & 98 \\
\hline $\begin{array}{l}\text { Calidad y } \\
\text { Equidad de la } \\
\text { Educación }\end{array}$ & 0 & $100 \%$ & $7 \%$ & $93 \%$ & $0 \%$ & $100 \%$ & $0 \%$ & $100 \%$ & 2 & 98 \\
\hline $\begin{array}{l}\text { Derechos } \\
\text { Humanos y } \\
\text { Ciudadanía }\end{array}$ & 33 & 67 & 20 & 80 & 20 & 80 & $20 \%$ & 80 & 23 & 77 \\
\hline $\begin{array}{l}\text { Diversidad } \\
\text { cultural }\end{array}$ & 47 & $53 \%$ & 40 & 60 & $0 \%$ & 100 & $0 \%$ & 100 & 22 & 78 \\
\hline $\begin{array}{l}\text { Aprendizaje } \\
\text { para toda la } \\
\text { vida }\end{array}$ & 0 & 100 & 13 & 87 & 0 & 100 & $0 \%$ & 100 & 3 & 97 \\
\hline $\begin{array}{l}\text { Desarrollo } \\
\text { Sostenible }\end{array}$ & 0 & 100 & 0 & 100 & 27 & 73 & $27 \%$ & 73 & 14 & 87 \\
\hline $\begin{array}{l}\text { Enfoque de } \\
\text { Género }\end{array}$ & 7 & 93 & 7 & 93 & 20 & 80 & $20 \%$ & 80 & 14 & 87 \\
\hline $\begin{array}{l}\text { Otras } \\
\text { (vulnerabilidad) }\end{array}$ & 7 & 93 & 20 & 80 & 20 & 80 & $20 \%$ & 80 & 17 & 83 \\
\hline $\begin{array}{l}\text { Promedio } \\
\text { General por } \\
\text { carrera }\end{array}$ & 13 & 87 & 13 & 87 & 10 & 90 & $10 \%$ & 90 & 12 & 89 \\
\hline
\end{tabular}

Fuente: elaboración propia

En este tercer ámbito, se muestra aquellos cursos sellos o transversales que las universidades incorporan de manera obligatoria u optativos a las mallas curriculares y que responden más a una formación en habilidades instrumentales que a saberes identitarios. Los datos son claros, en más del 80\% están ausentes las temáticas educativas ODS en más del 80\%.

Finalmente, está claro que en los textos programáticos y curriculares que proponen las universidades chilenas en la formación inicial del profesorado, no se están alineando con los ODS, y si lo hacen es solo en algunas temáticas o de forma parcial. 


\section{Discusión y conclusiones}

\section{Alineamiento de los proyectos FID con las temáticas educativas ODS}

Como se ha podido constatar, existe una narrativa que se alinea con las temáticas educativas ODS y están presentes en las propuestas curriculares de todas las pedagogías, en algunos casos como asignaturas en otros como contenidos específicos, más allá de que, como lo muestran los datos, su presencia en las propuestas FID no es reveladora (bajo el 50\%), especialmente en saberes relacionados al desarrollo sostenible y el enfoque de género, confirmando estudios que señalan que existe escaso conocimientos de los ODS.

También es bajo el número de profesores que poseen competencias de sostenibilidad ya que aún predomina una gestión tradicional, individualista, fragmentada y, poco proclive al trabajo colaborativo (De la Rosa Ruiz et al., 2019; Segado Segado et al., 2020). Esto porque se sigue en lógicas academicistas y homogéneas que no están dando cuenta de las necesidades educativas específicas de todos los estudiantes. (Bolívar, 2018; Magro, 2018;).

Los resultados muestran un alineamiento burocratizado de tendencia neoliberal (Espinoza, 2017) que ve a los estudiantes en formación inicial, continua o posgradual como consumidores. Por lo mismo, en los relatos no se observa una estrategia de alineamiento que tienda a los ODS, más bien sus acciones se orientan a responder con las exigencias sistémicas y sus políticas públicas (MINEDUC, 2016; Opazo et al., 2020). El supuesto es que, más allá de la existencia de un discurso institucional que las acoge, sus narrativas pro-ODS muestran que en la FID su presencia es al menos superficial. Por ejemplo, temáticas como el desarrollo sostenible solamente se sitúan en la educación ambiental, o en el enfoque de género donde aún prevalece la educación sexista que sigue naturalizado las desigualdades de género (Lapierre et al., 2019).

De acuerdo con lo expresado, no se puede concluir que la FID chilena se encuentre alineada con las temáticas educativas ODS, más bien, se puede explicar como un déficit o un alineamiento en proceso. Un segundo supuesto es que todo parecen ser respuestas adaptativas a las políticas públicas que presionan a las universidades a acomodarse con las nuevas agendas globales como en el caso de los ODS. Un tercer supuesto es que aún no se logra salir de la trinchera en la que predomina una formación curricular fragmentada, donde saberes cercanos al paradigma de la sostenibilidad o de una educación humanistas, inclusiva, de género, para la ciudadanía o democrática sigue estando más en la frontera de la formación docente que en el corazón o centro de ella (Martínez, 2020; Perrino-Peña, 2020; Salas Guzmán y Salas Guzmán, 2016).

\section{Integración curricular de las temáticas educativas ODS en la FID}

Transversalizar en el currículum temáticas como la democracia, la ciudadanía y los DDHH demanda superar visiones alojadas en la educación cívica y, su institucionalidad política (Cox, 2018; Magendzo y Arias, 2015). De ahí que la propuesta es consolidar una línea de formación humanista y para el desarrollo sostenible (Cuenca, 2020).

Con respecto a la educación inclusiva, que aparece como uno de los aprendizajes 
más críticos en la formación del profesorado, si bien está presente transversalmente en todos los casos investigados los estudios muestran un relato más retórico que práctico ya que tanto los estudiantes de pedagogía como los profesores en servicio se perciben sin las competencias para liderar y atender las necesidades educativas de todos los estudiantes, de las familias y sus comunidades educativas, más allá de la valoración que se tiene de la diversidad como fuente de aprendizaje (Cervera Delgado y Martí Reyes, 2018; Duk et al., 2019).

La propuesta es transitar de una formación homogénea a otras de las diferencias que supere currículos puramente técnicos, donde gane influencia el enfoque inclusivo en su rediseño curricular y en una formación práctica de calidad, equitativa, con base en la justicia y solidaridad (Herrera-Seda, 2018; Medina Sánchez, 2021; Martín et al., 2017; Valassina et al., 2019; Vélez et al., 2016;).

En este contexto la educación inclusiva viene a hacer el eje central del paradigma del desarrollo sostenible. Para Paz Delgado (2018) conlleva a un nuevo rol e identidad docente a partir de competencias colaborativas y reflexivas que se doten de técnicas y estrategias de adaptación de la enseñanza para todos.

\section{La perspectiva de los ODS para una nueva profesionalización docente}

Darling-Hammond (2008) y Marcelo y Vaillant (2018) plantean un agotamiento de la propuesta tradicional con la que se han formado los docentes y, que ha puesto en entredicho su núcleo curricular (Escudero, 2019). En ese marco la propuesta es pensar nuevos horizontes de profesionalización que impacte y renueve prácticas de enseñanza y aprendizaje inicial y en servicio. Partiendo por redefinir el diseño curricular en la FID, en este caso, bajo el paradigma que sostiene los ODS asociando sus procesos a aspectos claves como el género, los derechos humanos, la democracia y la ciudadanía, al desarrollo de competencia para la sostenibilidad, la inclusión educativa y la atención a la diversidad.

La FID en Chile está transitando a prácticas que se alinean con las temáticas educativas ODS más allá de no terminar de traducir esa mirada en diseños curriculares integrados, innovadores y profesionalizantes. El supuesto es que más que una ruptura con el modelo pedagógico tradicional serían respuestas adaptativas a demandas sistémicas. La propuesta entonces es resignificar la profesión docente poniendo el énfasis en sus dimensiones afectivas, reflexivas, de compromiso y contextualizadas (Martín-Romera y García-Martínez, 2018; Serrano Rodríguez yPontes Pedrajas, 2016).

En conclusión, se ha podido establecer que las universidades pedagógicas en su política institucional expresan una narrativa pro-ODS, no sucede lo mismo en los textos identitarios y curriculares (planes y programas) es más la integración de esa mirada muestra un alineamiento deficitario. Por lo mismo pareció un buen camino caracterizar el estado actual de los atributos que permiten identificar debilidades y oportunidades de transformación del modelo de gestión institucional y su alineamiento estratégico, como lo fue en el caso de la FID frente al paradigma educativo que representan los ODS, estableciendo indicadores de evaluación del desempeño del modelo formativo y curricular.

Nos deja este trabajo un conjunto de atributos basado en los ODS, tanto para definir 
la gestión universitaria como para otorgar posibilidades de mejora de la FID. En tal sentido lo que sigue es superar el carácter descriptivo y exploratorio de la investigación y ampliar a niveles de tipo longitudinal y comparativa; por ejemplo, confrontando dos periodos distintos de diseños curriculares que admita establecer tendencias en las dinámicas de adaptación universitaria. También la necesidad de considerar un mayor número de casos u otras estrategias como los métodos Delphi que se presentan como una buena opción.

Por último, las caracterizaciones del currículo de la FID en función de un modelo de alineamiento o adaptación a los ODS permiten anticipar programas de adecuación, profundización o complemento a los currículos actuales, pensando en formar profesores capaces de enfrentarse a un nuevo perfil de estudiantado, a nuevos desafíos didácticos, y a entornos sociales cambiantes y en conflicto. En ese sentido, el proceso y modelo de gestión universitaria tiene un espacio importante aún para ir adecuando sus programas a la perspectiva paradigmática que representan los ODS, como en el caso de este estudio.

\section{Referencias}

Ávalos, B. (2014). La formación inicial docente en Chile: Tensiones entre políticas de apoyo y control. Estudios Pedagógicos (Valdivia), 40(Especial), 11-28. https://dx.doi.org/10.4067/S0718-07052014000200002

Aznar Minguet, P.., Ull, M. Á., Piñero, A., yMartínez-Agut, M. P. (2017). La evaluación de la formación de formadores. Un catalizador en el proceso de cambio curricular hacia la sostenibilidad. Revista Iberoamericana de Educación(73), 225-252. https://doi.org/10.35362/rie730300

Bolívar, A. (2018). La colegialidad en la profesión docente. Déficits y caminos. Cuadernos de Pedagogía, 489, 46-51.

Castillo-Retamal, F., y Cordero-Tapia, F. (2019). La educación ambiental en la formación de profesores en Chile. UCMaule(56), 9-28. https://doi.org/10.29035/ucmaule.56.9

Cervera Delgado, C., y Martí Reyes, M. (2018). Formación docente para la inclusión y la diversidad: retos y agenda pendiente en México. Atenas, 3(43), 72-88. https://www.redalyc.org/articulo.oa?id=478055153005

Charmaz, K. (2013). La teoría fundamentada en el siglo XXI: Aplicaciones para promover estudios sobre la justicia social. Denzin, En: N. K.; (comps.), Y. S. Lincoln. Estrategias de investigación cualitativa: Vol. III., 270-325. Gedisa.

Cox, C. (2018, 23 de julio). Tres dimensiones de requerimientos a la formación inicial docente: competencias siglo 21, inclusión, ciudadanía. II Reunión Regional de Ministros de Educación de América Latina y el Caribe en Cochabamba-Bolivia. OREALC/UNESCO Santiago. http://www.unesco.org/new/fileadmin/ MULTIMEDIA/FIELD/Santiago/pdf/11-C-Cox-3-dimensiones-docente-ESP.pdf

CRES. (2009). Conferencia Nacional de la Educación Superior en América Latina y el Caribe. Declaraciones y plan de acción. Perfiles educativos, 31(125), 90-108. 
http://www.scielo.org.mx/scielo.php?script=sci_arttext\&pid=S018526982009000300007\&lng=es\&tlng=es.

Cuenca, R. (2020). Educación y democracía en America Latina: Un asunto de desarrollo sostenible. Profesorado, Revista de Currículum y Formación del Profesorado, 24(3), 27-47. https://doi.org/10.30827/profesorado.v24i3.13213

Darling-Hammond, L. (2008). The case for university-based teacher education. (S. S.N. In M. CochranSmith, Ed.) Handbook of Research search on Teacher Education, 333-346.

De la Rosa Ruíz. D., Giménez Armentia, P., \&De la Calle Maldonado, C. (2019). Educación para el desarrollo sostenible: el papel de la universidad en la Agenda 2030. Revista Prisma Social(25), 179-202. https://revistaprismasocial.es/article/ view/2709

Duk, C., Cisternas, T., y Ramos, L. (2019). Formación Docente desde un Enfoque Inclusivo. A 25 Años de la Declaración de Salamanca, Nuevos y Viejos Desafíos. Revista Latinoamericana de Educación Inclusiva, 13(2), 91-109. https://dx.doi.org/10.4067/S0718-73782019000200091

Escudero, J. M. (2019). La profesión y formación docente en discusión: cuál es el estado de la cuestión, qué cambiar y porqué, cómo habría de hacerse. En J. M. (Coord.)., Profesión y profesionalidad docente: Una acción educativa comprometida con el desarrollo humano (págs. 167-176). ANELE - REDE.

Espinoza, 0. (2017). Neoliberalismo y educación superior en Chile: una mirada crítica al rol desempeñado por el Banco Mundial y los "Chicago Boys". Laplage em Revista, 3(3), 93-114. http://dx.doi.org/10.24115/S2446-6220201733378p.93114

Fernández Batanero M. (2013). Competencias docentes y educación inclusiva. Revista Electrónica de Investigación Educativa, 15(2), 82-99. http://redie.uabc.mx/ vol15no2/contenido-fdzbatanero.html

Herrera-Seda, C. (2018). La Formación Inicial del Profesorado para una Educación Inclusiva: Desafíos, Oportunidades y Transformaciones. Revista latinoamericana de educación inclusiva, 12(2), 17-20. https://dx.doi.org/10.4067/S071873782018000200017

Lapierre, M., Ugueño, Á., Solar, F., Krause, A., Luna, L., Rilling, C., Fleet, A., Donoso, E. y García, G. (2019). Enfoques y Tendencias Actuales en Educación Superior Inclusiva. En M. Letelier (Ed.), Educación Superior Inclusiva (Primera ed., págs. 15-67). Santiago, Chile: Centro Interuniversitario de Desarrollo CINDA.

Llanos, G. M., y Solana, J. (13 de septiembre de 2016). IV Jornadas OCUD, sobre la cooperación universitaria al desarrollo retos en el nuevo escenario de la agenda de desarrollo 2015-2030. Documento de Partida para el proceso para la modificación de la Estrategia de Cooperación Universitaria al Desarrollo. Valencia, España: María Llanos Gómez. Universitat Politècnica de València Jorge Solana. Universidad de Alcalá. http://www.ocud.es/agenda2030/files/doc27/documento-de-partida.pdf

Magendzo, A. y Arias, R. (2015). Informe regional 2015: educación ciudadana y formación docente en países de América Latina.SREDECC: Editorial Gente Nueva. https://www.oas.org/cotep/GetAttach.aspx?lang=en\&cId=304\&aid=489 
Magro, M. C. (2018). Docentes para nuestra época. Cuadernos de Pedagogía, 489, 6371.

Marcelo, C., y Vaillant, D. (2018). La formación inicial docente: problemas complejos, respuestas disruptivas. Cuadernos de pedagogía, 489, 27-32.

Martín, C. S., Villalobos, C., Muñoz, C., y Wyman, I. (2017). Formación inicial docente para la Educación Inclusiva. Análisis de tres programas chilenos de pedagogía en Educación Básica que incorporan la perspectiva de la educación inclusiva. Calidad en la educación(46), 20-52. https://dx.doi.org/10.4067/S071845652017000100020

Martínez, L. M. (2020). Hacia una educación inclusiva: formación del profesorado de primaria enmarcada en los ODS que potencian la igualdad de género. Revista Iberoamericana de Educación, 2(82), 27-45.

Martín-Romera, A., y García-Martínez, I. (Enero-Marzo de 2018). Profesionalización del docente en la actualidad: contribuciones al desarrollo profesional. Profesorado: revista de currículum y formación del profesorado, 22(1). https://dx.doi.org/10.4067/S0718-45652017000100020

Medina Sánchez L. (2021). La formación inicial docente en educación inclusiva: experiencias y percepciones del futuro profesorado. REIDOCREA, 10(3), 1-24. http://dx.doi.org/10.30827/Digibug.66306

MINEDUC. (Agosto de 2016). Lineamientos de Políticas Públicas para Formación Inicial Docente. División de Educación Superior Ministerio de Educación. Santiago, Chile.

Moya Ureta, C. (2002). Integración, diversidad y ruptura. La pedagogía y la didáctica en la sociedad de la información. Material de clase Programa de Magíster en Pedagogía Universitaria. Talca, Chile: Universidad Católica del Maule.

NACIONES UNIDAS. (2015). Transformar nuestro mundo: la Agenda 2030 para el Desarrollo Sostenible. https://unctad.org/meetings/es/SessionalDocuments/ ares70d1_es.pdf

Noguero, F. L. (2002). El análisis de contenido como método de Investigación. ${ }^{\circledR} X X I$, Revista de Educación(4), 167-179.

Opazo, H., Castillo, J., y Carreño, Á. (2020). Los desafíos de la meta 4.7 de la agenda 2030: Un análisis de evidencias desde UNESDOC. Profesorado, Revista de Currículum y Formación del Profesorado, 24(3), 49-73. https://doi.org/10.30827/profesorado.v24i3.15402

Paz Delgado, C. (2018). Formación inicial de docentes para la atención a la diversidad en contextos inclusivos. Paradigma: Revista De Investigación Educativa, 24(37), 32-47. https://doi.org/10.5377/paradigma.v24i37.6512

Perrino-Peña, M., y González-Palomares, A. (4 de dic. de 2020). Género y planes de estudio: Análisis del Grado en Ciencias de la Actividad Física y del Deporte. Profesorado, Revista de Currículum y Formación del Profesorado, 24(3), 291-308. https://doi.org/10.30827/profesorado.v24i3.8151

Portales, P. (comp). (2016). Perspectivas sobre políticas docentes en América Latina y el Caribe. Aprendizajes de la Estrategia Regional de Docentes de la OREALC/UNESCO 2012-2016. Oficina Regional de Educación para América Latina y el Caribe (OREALC/UNESCO Santiago). 
Rodríguez García, G., Flores Gil J., y García Jiménez, E. (1999). Metodología de la investigación cualitativa. (2 ${ }^{\mathrm{a}}$ ed.). Aljibe.

Salas Guzmán, N. y Salas Guzmán, M. (2016). Tiza de Colores: Hacia la Enseñanza de la Inclusión sobre Diversidad Sexual en la Formación Inicial Docente. Revista latinoamericana de educación inclusiva, 10(2), 73-91. https://dx.doi.org/ 10.4067/S0718-73782016000200006

Sánchez, J. (2021). Marcos interpretativos de género en los estándares. En S. Larraín, \& G. Guajardo (Edits.), Niñez y Género: Claves de comprensión y acción. CIDENI, FLACSO-Chile.

Segado Segado, I.; Sánchez Vidal, M. E., y Hernández Aguado, Simón. (2020). Integración de los Objetivos de Desarrollo Sostenible en la UPCT: Aproximación a nivel curricular. CUADERNOS CEDE 2030, Cátedra de Cultura y Ética Directiva y Empresarial. UPCT. Universidad Politécnica de Cartagena. http://hdl.handle.net/10317/8413

Serrano Rodríguez, R. y Pontes Pedrajas, A. (2016). El desarrollo de la Identidad Profesional Docente en la formación inicial del profesorado de secundaria. Enseñanza \& Teaching, 3555.http://dx.doi.org/10.14201/et20163413555UNESCO - IIEP. (2017). Seis maneras de asegurar que la Educación Superior no deje a nadie atrás. (30), Documento de Política, $10 \quad$ p. http://unesdoc.unesco.org/images/0024/002478/247862S.pdf

UNESCO. (2016). Educación 2030: Declaración de Incheon y Marco de Acción para la realización del Objetivo de Desarrollo Sostenible 4: Garantizar une aducación inclusiva y equitativa de calidad y promover oportunidades de aprendizaje permanente para todos. 86. Biblioteca Digital de la UNESCO. https://unesdoc.unesco.org/ark:/48223/pf0000245656_spa.locale=es

UNESCO. (2017). CCONG 2017/Educación 2030: Consecución del ODS 4-Educación 2030, declaración de la reunión mundial. Siem Reap, 8 y 9 de mayo, Camboya. https://unesdoc.unesco.org/permalink/P-d1965fbf-beb3-415a-8e4ad809cabec84d

Valassina, F., Letelier, P., Letelier, M., Angulo, J., Faúndez, F., Márquez, E. S., y Valverde, M. (2019). Orientaciones para una política institucional de formación inclusiva en las universidades chilenas. En Educación Superior Inclusiva (págs. 103-171). Santiago, Chile: Centro Interuniversitario de Desarrollo CINDA.

Vásquez, C., y García-Alonso, I. (4 de dic. de 2020). La educación estadística para el desarrollo sostenible en la formación del profesorado. Profesorado. Revista de Curriculum y Formación del Profesorado, 24(3), 125-147. https:// doi.org/ 10.30827/profesorado.v24i3.1521

Vilches, A. C., Martínez, M., Mayoral, O., Ull, A., y Vázquez, V. (2018). Atención a la Sostenibilidad y los Objetivos de Desarrollo Sostenible (ODS) en la formación del profesorado. Un ejemplo de puentes entre la investigación y la práctica. VI Seminario Iberoamericano CTS, X Seminario Ibérico CTS, 1-3 de agosto 2018. Buenos Aires, Argentina: Universidad de Quilme. 